\title{
The role played by thermal feedback in heated Farley-Buneman waves at high latitudes
}

\author{
J.-P. St.-Maurice, R. S. Kissack \\ Department of Physics and Astronomy, The University of Western Ontario, London, Ontario N6A 3K7, Canada
}

Received: 14 December 1998 / Revised: 23 November 1999 / Accepted: 3 December 1999

\begin{abstract}
It is becoming increasingly clear that electron thermal effects have to be taken into account when dealing with the theory of ionospheric instabilities in the high-latitude ionosphere. Unfortunately, the mathematical complexity often hides the physical processes at work. We follow the limiting cases of a complex but systematic generalized fluid approach to get to the heart of the thermal processes that affect the stability of $E$ region waves during electron heating events. We try to show as simply as possible under what conditions thermal effects contribute to the destabilization of strongly field-aligned (zero aspect angle) Farley-Buneman modes. We show that destabilization can arise from a combination of (1) a reduction in pressure gradients associated with temperature fluctuations that are out of phase with density fluctuations, and (2) thermal diffusion, which takes the electrons from regions of enhanced temperatures to regions of negative temperature fluctuations, and therefore enhanced densities. However, we also show that, contrary to what has been suggested in the past, for modes excited along the $\mathbf{E}_{0} \times \mathbf{B}$ direction thermal feedback decreases the growth rate and raises the threshold speed of the Farley-Buneman instability. The increase in threshold speed appears to be important enough to explain the generation of 'Type IV' waves in the high-latitude ionosphere.
\end{abstract}

Key words: Ionosphere (auroral ionosphere; ionospheric irregularities; plasma waves and instabilities)

\section{Introduction}

In the classical treatment of the Farley-Buneman instability mechanism (e.g., Fejer et al., 1984) the electrons

Correspondence to: J.-P. St.-Maurice are considered isothermal, mostly for simplicity. However, this simplification can lead to many difficulties when faced with an interpretation of irregularity data, particularly those coming from coherent radars. For instance, it is becoming widely accepted that large amplitude waves are often observed with a mean Doppler shift that matches the threshold speed predicted by linear instability theory. Even the different "types" that have mean Doppler shifts that are markedly different from the nominal ion-acoustic speed of the medium have been linked to threshold conditions through the influence of gradients (e.g. St.-Maurice et al., 1994) or through electron heating effects (e.g. Farley and Providakes, 1989; Shalimov and Haldoupis, 1995).

Naturally, given the many exceptions to the rule of large amplitude waves with Doppler shifts equal to the nominal ion-acoustic speed of the medium at small flow angles, an increasingly large number of researchers have raised questions about the validity of the isothermal assumption that has been used to calculate this ionacoustic speed. For example, Farley and Providakes (1989) proposed that at low enough frequencies the electrons should be considered adiabatic rather than isothermal, which would lead to an increase in the ionacoustic speed for the problem at hand. However, in order to get adiabatic results these authors had to assume that the frequencies were low enough for heat conduction not to be an important factor, and they had to neglect the effects of the heating and cooling rates on the perturbed electron energy balance.

In a different vein, Pécseli et al. (1989) and Gurevich and Karashtin (1984) respectively pointed out that electron thermal conduction and electron thermal diffusion should be included for the treatment of waves a few meters in size and greater. However, Pécseli et al. (1989) neglected the variations in the electron collision frequency with temperature (and hence, thermal diffusion effects) and they did not include the influence of perturbed heating and cooling rates. As for the thermal diffusion work, starting with the physics used by 
Gurevich and Karashtin (1984), Shalimov and Haldoupis (1995) argued that in the presence of electron heating events observed under strong $(>40 \mathrm{mV} / \mathrm{m})$ electric field conditions, thermal diffusion could affect the waves in such a way as to markedly decrease the threshold speed of the instability, even for $m$ size waves. However, Shalimov and Haldoupis (1995) neglected ion inertia in a part of their numerical calculations, which we will show led to much greater thermal diffusion effects than should have been expected.

More recently, Dimant and Sudan $(1995,1997)$ and Robinson (1998) pointed out that ordinary electron Pedersen conductivity, through perturbed Joule (ohmic) heating effects, could further destabilize low frequency waves at flow angles between the Hall and Pedersen flow directions. Along the same lines, Kissack et al. (1995, 1997) used the fact that low frequency waves should be describable with fluid theory in order to evolve a systematic fluid framework of the Farley-Buneman instability based on Grad's 8-moment approximation, to produce a theory that included electron energy and heat flow effects. One difference with the Dimant and Sudan work was that they chose to cast their results in a form that offered a clear generalization of the classical treatment. A second difference was that Kissack et al. $(1995,1997)$ allowed for an arbitrary behavior of the electron-neutral interactions so that the elastic collision frequency and the inelastic energy exchange rates were given in terms of arbitrary functions of $T_{e}$, not just power laws. Finally, Kissack et al. $(1995,1997)$ have, as we shall see in more detail here, a more general treatment of electron heating which also allows them to study situations for which the electron temperature is much greater than the neutral temperature.

In order to better guide the reader, we start our presentation by first giving an overview of the physics at work before going through more detailed mathematical derivations. We hope in this way to make the rest of the work easier to follow since the reader will know what we are after from the very beginning. Therefore, after a presentation focused on the physics (Sect. 2), we discuss in Sect. 3 the individual contributions from the perturbed energy and heat flow equations. We show in the process that some improvements must be brought to the Shalimov and Haldoupis (1995) treatment of the algebra, and discuss the implications in Sect. 4.

\section{Physical processes}

\subsection{Classical mechanisms}

The starting point in the description of $E$ region irregularities is the fact that electrons basically $\mathbf{E}_{0} \times \mathbf{B}$ drift while ions only have a small Pedersen drift to leading order. Thus, when a positive density perturbation is created in the medium, the electrons rush ahead in the $\mathbf{E}_{0} \times \mathbf{B}$ direction, while the ions at first stay put. The result is a polarization electric field in the $\mathbf{E}_{0} \times \mathbf{B}$ direction in regions of density enhancements and vice- versa for negative density perturbations. In response to this new (perturbed) field, Pedersen currents borne mostly by the ions will start flowing in the $\mathbf{E}_{0} \times \mathbf{B}$ direction. These currents will limit the strength of the polarization field by requiring a quick stop to the growth in the net charge density, that is, by creating a null current divergence situation. This requirement enables one to relate the perturbed density fluctuation to the perturbed polarization field. To leading order this balance is obtained by neglecting pressure gradient effects and inertia so that the perturbed total current, $\delta \mathbf{J}$, is taken to be basically in the $\mathbf{E}_{0} \times \mathbf{B}$ direction and to be simply given by

$$
\frac{\delta \mathbf{J}}{e} \approx n_{0}\left(\frac{\Omega_{i}}{v_{i}}+\frac{v_{e}}{\Omega_{e}}\right) \frac{\delta \mathbf{E}}{B}-\delta n \frac{\mathbf{E}_{0} \times \mathbf{B}}{B^{2}}
$$

where $\delta$ stands for perturbed quantities and the subscript 0 describes the zeroth order terms. In addition, $\mathbf{E}$ and $\mathbf{B}$ are the electric and magnetic fields, $n$ is the density, and $\Omega_{i}$ and $v_{i}$ are the ion cyclotron and ionneutral collision frequencies respectively, with a similar notation for the electrons. In the $E$ region, for electrons as well as for ions, only collisions with neutrals need to be considered. The approximate balance that we just expressed is only valid, of course, at long enough wavelengths so that diamagnetic currents related to the pressure perturbations can be neglected. In practice, at $115 \mathrm{~km}$ at high latitudes, and for electrons $\mathbf{E}_{0} \times \mathbf{B}$ drifts of the order of $1000 \mathrm{~m} / \mathrm{s}$, this means wavelengths of the order of $2 \mathrm{~m}$ or longer (see Kelley, 1989, for details regarding this determination).

Imposing next a null divergence in the perturbed current gives the important leading order relation

$$
\frac{\delta E}{B}=\frac{v_{i}}{\Omega_{i}} \frac{1}{1+\psi} V_{e 0} \cos \theta \frac{\delta n}{n_{0}}
$$

where $V_{e 0}$ is the magnitude of the electrons $\mathbf{E}_{0} \times \mathbf{B}$ drift, and $\theta$ is the 'flow angle' between the $\mathbf{E}_{0} \times \mathbf{B}$ drift direction and the direction of the wave-vector (or perturbed electric field). Also, for null aspect angles (pure two-dimensional situations) $\psi=v_{e} v_{i} / \Omega_{e} \Omega_{i}$ is the ratio of the electron to ion Pedersen currents and, consistent with the statement at the beginning of this section, is normally taken to be smaller than 1 in the $E$ region.

It is important to note that Eqs. (1) and (2) provide leading order balances that include neither the inertial (destabilizing) effects nor the pressure gradient (diffusive, stabilizing) terms that have an influence on the longer time scale evolution of the initial structure. As a result, if one uses Eq. (2) in the continuity equation of, say, the ions, one gets a very well-known expression for the phase velocity of the structures, which is given by

$v_{p h}=\frac{V_{e 0} \cos \theta}{1+\psi}$

We are only interested here in changes in the amplitudes of the modes, which involve the slowly operating feedback and diffusive mechanisms responsible for wave growth or decay. We will therefore not consider changes 
to result 3 in the rest of the work and simply refer to Eq. (3) as a good approximation, in general, for the phase velocity.

Where things really vary from one treatment of the instabilities to another is with the feedbacks that slowly (in comparison to the time it takes to get result 2) affect the amplitude of the initial perturbation. For the classic Farley-Buneman wave situation, ion inertia is the key to creating a growth condition: the ambient ion response is delayed somewhat when they see the electrostatic structure given by Eq. (2) moving with the speed given by Eq. (3). As a result, some of the ions that were supposed to discharge positive structures (and hence regions of enhanced plasma densities) end up on the positive side of the moving irregularity and add to the charge of the perturbation instead. The perturbation then grows by requiring that charge neutrality be maintained in spite of the new addition. The resulting growth factor must be fast enough, however, to overtake the stabilizing influence of ordinary diffusion. Otherwise, the initial perturbation still decays.

More formally, the Farley-Buneman results are obtained by writing down the electron and ion continuity equations:

$\left[\frac{\partial}{\partial t}+\mathbf{V}_{e 0} \cdot \nabla\right] \delta n=-n_{0} \nabla \cdot \delta \mathbf{v}_{e}$

$\frac{\partial \delta n}{\partial t}=-n_{0} \nabla \cdot \delta \mathbf{v}_{i}$

where we have assumed that the zeroth order ion drift is negligible and that the derivatives of $n_{i}$ and $n_{e}$ are the same because the net charge does not change over the long time scale of interest here.

To keep things at their simplest, we will neglect parallel current perturbations throughout this work. We will also introduce the thermal effects in gradual fashion. First we will cover the standard isothermal situation (this subsection), then introduce only temperature variations in relation to the pressure gradient effects (next subsection) before considering additional effects due to the temperature dependence of the collision frequency (thermal diffusion and thermoelectric effects, to be discussed for the rest of the work).

In terms of pressure fluctuations (which could include temperature fluctuations under the right circumstances), but not in terms of temperature dependent collision frequencies, the perturbed electron velocity depends only on a perturbed electric field term and a perpendicular pressure gradient term such that the electron velocity divergence needed in Eq. (4) is given by

$\nabla \cdot \delta \mathbf{v}_{e}=D_{e}\left[\nabla_{\perp}^{2} \frac{e \delta \phi}{T_{e 0}}-\nabla_{\perp}^{2} \frac{\delta p_{e}}{p_{e 0}}\right]$

where $D_{e}=v_{e} T_{e 0} / m_{e} \Omega_{e}^{2}$ is the electron diffusion coefficient and where $\phi$ is the electrostatic potential, $e$ is the magnitude of the electronic charge, $m_{e}$ is the electron mass, $T_{e}$ is the electron temperature expressed in energy units, and $p_{e}$ is the electron pressure. The relation between the perturbed potential $\nabla \delta \phi=-\delta \mathbf{E}$ and the perturbed densities can be obtained from the ion continuity and momentum equations, as per the standard treatment. The result is the well-known expression

$\nabla \cdot \frac{\delta \mathbf{E}}{B}=k^{2} \frac{\delta \phi}{B}=\frac{1}{\Omega_{i}} \frac{\delta n}{n_{0}}\left(\omega^{2}-k^{2} \frac{T_{i 0}}{m_{i}}+i \omega v_{i}\right)$

where we are now switching to a plane wave analysis notation in time and space and where $\omega$ is the (complex) frequency. Using the last two equations and assuming that the pressure fluctuations are only due to density fluctuations (with isothermal electrons and ions) we get the well-known dispersion relation

$\omega-\mathbf{k} \cdot \mathbf{V}_{e 0 \perp}+\frac{\psi}{v_{i}}\left\{i k_{\perp}^{2}\left(\frac{T_{i 0}}{m_{i}}+\frac{T_{e 0}}{m_{i}}\right)+\omega\left(v_{i}-i \omega\right)\right\}=0$

It is easy to see from this that, for $\psi$ small enough, the root for the frequency is real to leading order. In that context, the $\omega^{2}$ term (ion inertia) and the $k_{\perp}^{2}$ terms (ambipolar diffusion) can be viewed as corrections to a leading order expression for the frequency (or the phase velocity $\omega_{r} / k$ ) given by Eq. (3). This is the classic Farley-Buneman result. Note that the diffusion and ion inertia corrections to the dispersion relation work in opposite directions in that case and that the amplitude grows if the inertial term is large enough to overcome the stabilizing effects of diffusion.

The second classic destabilizing factor that has been well-studied is the gradient-drift mechanism. This mechanism has to be invoked when the wavelengths are so long that ion inertia contributes very little to the growth of the structure. In that case an ambient density gradient is able to affect the structure a lot more easily than ion inertia. The effect of the density gradient comes from a modification of the electron continuity equation in the presence of an ambient density gradient, which now gives the relation

$\left[\frac{\partial}{\partial t}+\mathbf{V}_{e 0} \cdot \nabla\right] \delta n=-n_{0} \nabla \cdot \delta \mathbf{v}_{e}-\delta \mathbf{v}_{e} \cdot \nabla n_{0}$

Without having to write the final dispersion relation, we can see how the new term on the right-hand-side will affect the results: imagine, to keep things simple, that the perturbed electric field appearing in Eq. (1) points in the $\mathbf{E}_{0} \times \mathbf{B}$ direction for positive density perturbations. However, recall that the electrons are strongly magnetized, and that the wave frequency is much lower than the electron gyrofrequency (and the wavelength much greater than the electron gyroradius). Consequently, the electrons' dominant response to the electric field of the structure is to acquire a $\delta \mathbf{E} \times \mathbf{B}$ drift against the original electric field direction. However, if the ambient density increases in the same direction as the original field, we are then bringing a surplus of electrons through the presence of the $\delta \mathbf{E} \times \mathbf{B}$ drift, which creates the $-\delta \mathbf{v}_{e} \cdot \nabla n_{0}$ effect in Eq. (9). Once these new electrons are brought in, the fast time scales that lead to null current divergences once again take over and the net result is an increase in the original density perturbation. 
There is then a net growth in the structure, provided, once again, that the rate is fast enough to overcome ordinary diffusion.

\subsection{Thermal effects from the ordinary pressure term: the physics behind the Pedersen conductivity instability}

In this work we wish to emphasize the next level of complexity, which comes from thermal effects. In both the classical Farley-Buneman and gradient-drift treatments, the electron temperature perturbations are normally neglected (isothermal assumption). We will correct this situation by examining the temperature perturbations of electrons only. The electron temperature fluctuations are by far the most important because their small mass, compared to that of the neutrals, allows them to be cooled less easily than the ions and to have relatively large temperature fluctuations as a result. These fluctuations are then felt by the whole plasma through the usual polarization fields.

There are two ways through which temperature fluctuations can affect the results. One is through the contribution of temperature fluctuations to the pressure gradient fluctuations. Another is through the temperature dependence of the electron collision frequency, which creates thermal diffusion effects. Let us first neglect thermal diffusion and consider the pressure term alone. We already wrote the expression we need for the perturbed electron momentum as a function of pressure gradient and perturbed potential in Eq. (6). Only in this case, we keep the electron temperature perturbations in the pressure perturbation term. It is therefore easy to show that this implies that the diffusion operator is now given by

$\frac{\psi}{v_{i}} C_{s}^{2} \nabla^{2}\left(\frac{\delta n}{n_{0}}\right) \longrightarrow \frac{\psi}{v_{i}} C_{s}^{2}\left[\nabla^{2}\left(\frac{\delta n}{n_{0}}\right)+\frac{T_{e 0}}{T_{e 0}+T_{i 0}} \nabla^{2}\left(\frac{\delta T_{e}}{T_{e 0}}\right)\right]$

where $C_{s}^{2}=\left(T_{i 0}+T_{e 0}\right) / m_{i}$ is the square of the ionacoustic speed and energy units are still being used here for the temperatures. We should observe from Eq. (10) that thermal effects can contribute either to diffusive or to 'antidiffusive' processes. For example, consider a simple adiabatic situation for which $\delta n$ and $\delta T$ would increase together in response to compressional effects. The temperature fluctuations would then contribute to diffusion in much the same way the density fluctuations would (thermal fluctuations in phase with density fluctuations), thus making the plasma more stable. The key for thermal instabilities in general, and the 'Pedersen conductivity instability' (Robinson, 1998) in particular, comes from being able to do the opposite, namely, introducing temperature fluctuations that are out of phase with the density fluctuations. In the Pedersen conductivity instability case this is done most efficiently when the wave vector makes a $45^{\circ}$ angle with respect to the $\mathbf{E}_{0} \times \mathbf{B}$ direction.

Our quick discussion of the Pedersen conductivity instability is based on Robinson's (1998) treatment, and his paper can be consulted for details. The only thing we wish to stress here is the link of the instability with thermal fluctuations driven by electron ohmic dissipation (that is, electron frictional heating). We will only deal with the instability in its simplest form, which is the situation described by Robinson (1998) since it contains all of the basic driving physics (see Dimant and Sudan, 1995, 1997, for a much more general treatment).

To get the basic Pedersen instability result, it is best to examine the electron energy equation first. It can be written as

$$
\begin{aligned}
\frac{3}{2} & \frac{D}{D t}\left\{\ln \left(\frac{p_{e}}{n_{e}^{5 / 3}}\right)\right\}=\frac{3}{2} n \frac{D T_{e}}{D t}-T_{e} \frac{D n}{D t} \\
& =-\nabla \cdot \mathbf{q}_{e}-n_{e} \eta\left(T_{e}-T_{n}\right)+n_{e} \epsilon v_{e} m_{e}\left|\mathbf{v}_{e}\right|^{2}
\end{aligned}
$$

where the convective derivative $D / D t$ is equal to $\left(\partial / \partial t+\mathbf{v}_{e} \cdot \nabla\right)$ and $\mathbf{q}_{e}$ is the electron heat flow. Also, following Robinson's notation, we have written the cooling rate as $C_{e}=n_{e} \eta\left(T_{e}-T_{n}\right)$ where $T_{n}$ is the temperature of the neutral gas and $\eta$ is a coefficient that we will assume for the moment to depend only weakly on temperature. The frictional (or ohmic) heating rate is given by the term $Q_{e}=n_{e} v_{e} m_{e} \epsilon v_{e}^{2}$ where $m_{e}$ is the electron mass and $\mathbf{v}_{e}$ is the electron drift. The parameter $\epsilon$ (which requires some discussion, as is done later in the present work) is a parameter introduced by Robinson (1998) to allow the heating rate to be increased by wave electric fields in the turbulent plasma. Without such a contribution, one simply uses $\epsilon=1$, as was done by Dimant and Sudan (1995).

We assume next that energy transport effects are of secondary importance both at the zeroth order level and at the perturbed level. This means that the electron heating rate equates the electron cooling rate to zeroth order. At the perturbed level it also means that we are, strictly speaking, dealing with low frequency, long wavelength waves.

Given that both the heating and cooling rates are normally assumed to be proportional to the density, and given that we assume the heating and cooling rates to balance at the zeroth order level, the perturbed energy equation does not involve perturbed densities. We can then obtain a simple relation between temperature fluctuations and velocity fluctuations, which it is easy to show from Eq. (11), is given by:

$\frac{\delta T_{e}}{T_{e 0}}=\frac{2 \epsilon v_{e} m_{e} \mathbf{V}_{e 0} \cdot \delta \mathbf{v}_{e}}{\eta T_{e 0}}$

Recall, however, that $\delta \mathbf{v}_{e}$ is by far dominated by the electron $\delta \mathbf{E} \times \mathbf{B}$ drift through the structure. Defining our directions as shown in Fig. 1 (with $\theta$ positive in the $\left[-\mathbf{E}_{0},+\mathbf{E}_{0} \times \mathbf{B}\right]$ quadrant), this means that if the wavevector makes a flow angle between 0 and $90^{\circ}$, the dot product given in Eq. (12) is negative. As a result, the temperature fluctuations are negative. The reason is that the magnitude of the total drift (background plus fluctuation) is smaller than that of the ambient drift, so that the temperature is smaller (less friction) than the 


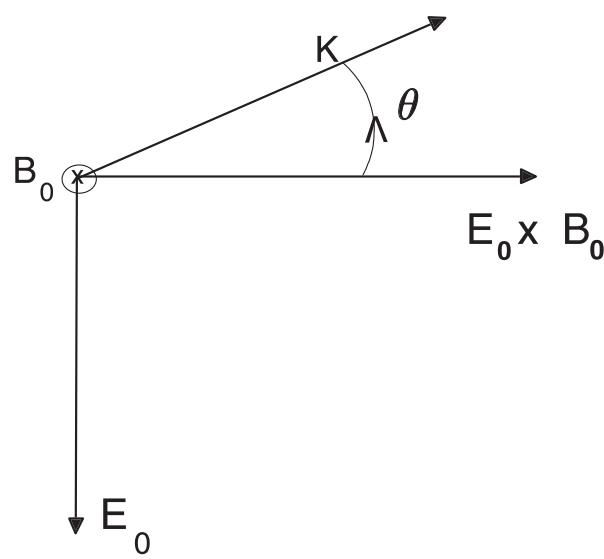

Fig. 1. Coordinate system being used to describe the angle $\theta$ in the Pedersen conductivity instability

average ambient temperature. This state of affairs is simply described by the equation

$\mathbf{V}_{e 0} \cdot \delta \mathbf{v}_{e} \simeq-V_{e 0} \sin \theta \frac{\delta E}{B}$

We can now use Eq. (2) to relate the perturbed field to the perturbed density to leading order. It is easy to show that this gives a temperature fluctuation which is anticorrelated with the density fluctuations for wavevectors between 0 and $+90^{\circ}$ to the $\mathbf{E}_{0} \times \mathbf{B}$ direction. The pressure fluctuation is therefore reduced by the waves and in that sense, through this negative effect, the plasma is rendered more unstable. Using Eqs. (10), (12), and (13), it is as if the ion-acoustic speed of the medium, which is responsible for diffusion, was reduced by having

$C_{s}^{2} \longrightarrow C_{s}^{2}-\epsilon V_{e 0}^{2} \frac{\Omega_{i}}{\eta} \frac{\psi}{1+\psi} \sin (2 \theta)$.

This shows that the Pedersen conductivity instability is simply driven by temperature fluctuations that are anticorrelated with the density fluctuations in the very pressure fluctuation term that normally leads to diffusion. The $180^{\circ}$ phase difference is in turn due to a decrease in the electron temperature when the velocity fluctuations are such as to decrease to net relative speed between electrons and neutrals, thereby reducing the amount of frictional heating at that particular point. Finally, we have to stress the existence of a typo in Robinson (1998), who showed a sign opposite to our final result Eq. (14), but only in his final equation.

\subsection{Thermal diffusion as an amplifying mechanism}

A higher level of complexity occurs with thermal feedbacks when we take the temperature dependence of the electron collision frequency into account. 'Thermal diffusion' effects are one of the results. Thermal diffusion affects the electron motion through the introduction of a heat flow term in the momentum equation. Likewise, one also finds a momentum term in the heat flow equation. When everything is said and done, when compared to Eq. (6), the divergence of the electron momentum ends up with two additional terms, one of which is a diffusion term driven by temperature fluctuations alone. That is to say, we now get

$$
\begin{aligned}
\nabla \cdot \delta \mathbf{v}_{e}= & D_{e}\left[\nabla_{\perp}^{2} \frac{e \delta \phi}{T_{e 0}}-\nabla_{\perp}^{2} \frac{\delta p_{e}}{p_{e 0}}-g \nabla_{\perp}^{2} \frac{\delta T_{e}}{T_{e 0}}\right] \\
& -g \frac{v_{e}}{\Omega_{e}} \frac{\mathbf{E}_{0}}{B} \cdot \nabla \frac{\delta T_{e}}{T_{e 0}}
\end{aligned}
$$

where $g$ is proportional to the derivative of the collision frequency, that is,

$g=\left[\frac{T_{e 0}}{v_{e}} \frac{\partial v_{e}}{\partial T_{e}}\right]_{T_{e 0}}$.

The value of $g$ is often taken to be $5 / 6$ for the electron temperatures of interest here (more on this later). Because of our present focus on thermal diffusion effects alone, we will not deal here with the Pedersen drift term in $v_{e} / \Omega_{e}$ more than by merely noting its presence. We will, on the other hand, go into considerably more details in the next section on the origin of the thermal diffusion term and its role.

One immediate consequence of the new temperature fluctuation term in Eq. (15) is the necessary amplification of the Pedersen conductivity instability effect. This is easy to see since the temperature terms associated with diffusion are obviously being multiplied by a factor $(1+g)$ if we compare the temperature contributions from the diffusion terms between Eqs. (15) and (6). As a result, for the Pedersen conductivity instability we must have

$$
C_{s}^{2} \longrightarrow C_{s}^{2}-(1+g) \epsilon V_{e 0}^{2} \frac{\Omega_{i}}{\eta} \frac{\psi}{1+\psi} \sin (2 \theta) .
$$

Therefore, thermal diffusion amplifies the effects of the temperature fluctuations by close to a factor of 2 . However, we must stress for completeness that if we were to use classical frictional heating alone $(\epsilon=1)$, we would find that the last term in Eq. (15) also contributes appreciably to a reduction of the effective ion acoustic speed through an electron temperature fluctuation term which is $90^{\circ}$ out of phase with the density fluctuation. In effect we would then have $\epsilon \rightarrow \epsilon+[(1+\psi) g / 2]$, which is, however, very close to $\epsilon$ for the expected $\epsilon=O(10)$ situation during electron heating events at high latitudes. Incidentally, our upper limit of about 10 or less for $\epsilon$ is based on the fact that the heating rate appears to normally be somewhat less than one order of magnitude greater than what can be attributed to ordinary frictional heating (e.g., St.-Maurice, 1987, 1990). The work of Robinson (1986) would produce perhaps twice as much because, by not being concerned with constraints on broadband density and electric fields like St.-Maurice $(1987,1990)$ was, he did not see a need to cut the cooling rates down by a factor of 2 as St.-Maurice had to do repeatedly.

Let us now go back to a situation for which there can be no Pedersen conductivity instability, namely one for which the wave vector is aligned with the $\mathbf{V}_{e 0}$, or $\mathbf{E}_{0} \times \mathbf{B}$ direction. It has been suggested (e.g., Shalimov and 
Haldoupis, 1995) that thermal diffusion could nevertheless, by itself, be an important destabilizing factor rather than just an amplification factor in that case. While the calculations are rather complex and will therefore have to be covered separately in some detail in the next section, we wish to make a few key points here so as to at least guide the reader's mind. A first observation comes from writing the perturbed energy Eq. (11) in the following form:

$\frac{3}{2} \frac{D \delta T_{e}}{D t} \approx-T_{e 0} \nabla \cdot \delta \mathbf{v}_{e}-\frac{\nabla \cdot \delta \mathbf{q}_{e}}{n_{e 0}}-\eta \delta T_{e}$.

It can be seen that there is no perturbed heating term in this equation. This is because $\mathbf{V}_{e 0} \cdot \delta \mathbf{v}_{e}$ is assumed to be small in the $\mathbf{E}_{0} \times \mathbf{B}$ direction and $\epsilon$ should be less than 10. Furthermore, there are no perturbed density term in Eq. (18) because (1) we are assuming that frictional heating balances the cooling term to zeroth order during heating events and also because (2) we assume that the nonlinear wave heating rates responsible for the heating are an enhancement in the Joule heating rate, which means that they are proportional to the electron density.

Unfortunately, a determination of the temperature perturbation becomes a complicated affair from this point on. The left-hand-side of Eq. (18), as well as the velocity divergence, heat flow term and cooling term all have contributions that contain a temperature fluctuation effect. We show in Sects. 3 and 4 that the results can nevertheless be expressed in a manner that looks very much like the familiar-looking dispersion relation given by Eq. (8), namely,

$$
\begin{aligned}
\omega & -\mathbf{k} \cdot \mathbf{V}_{e 0 \perp}+\frac{\psi}{v_{i}}\left\{i k_{\perp}^{2} \frac{T_{i 0}}{m_{i}}\right. \\
& \left.+i k_{\perp}^{2} \frac{T_{e 0}}{m_{i}}\left[1+(1+g) \frac{\delta T_{e} / T_{e 0}}{\delta n / n_{0}}\right]+\omega\left(v_{i}-i \omega\right)\right\}=0 .
\end{aligned}
$$

Our point here is that unlike the simpler Pedersen instability case where the temperature fluctuation is easily found in terms of Perturbed Joule heating effects, the task of evaluating $\delta T_{e} / \delta n$ is far more complicated here. We show in Sect. 4 and in the Appendix that when the wave vector is parallel to the $\mathbf{E}_{0} \times \mathbf{B}$ drift (as in the present case) the real part of the temperature to density fluctuation ratio is actually positive when the growth rate is positive. We can then see from Eq. (19) that all thermal feedback terms in this case, which include thermal diffusion, can only make the plasma more weakly unstable by amplifying the effects of ordinary diffusion.

\subsection{How thermal diffusion amplifies temperature fluctuations}

We have seen that thermal diffusion enhances the role played by diffusion through the factor $g$ in $(1+g)$. As we already indicated this is equivalent to having the ionacoustic speed $C_{s}$ replaced by the speed $C_{s}^{*}$ given by
$C_{s}^{* 2}=\frac{T_{i 0}}{m_{i}}+\frac{T_{e 0}}{m_{i}}\left[1+(1+g) \operatorname{Re}\left(\frac{\delta T_{e} / T_{e 0}}{\delta n / n_{0}}\right)\right]$.

We recall that this result is not general. As discussed in Sect. 2.3, additional Pedersen drift-related terms are present if the $\mathbf{k}$ vector is rotated away from the $\mathbf{E}_{0} \times \mathbf{B}$ direction.

Still, there is plenty of physics to discuss in relation to the thermal diffusion contribution presented in Eq. (20). To start with, it is very obvious that when there are no temperature fluctuations, the results are isothermal and thermal feedback plays no role. However, when the ratio $\delta T_{e} / \delta n$ is positive, thermal diffusion enhances the diffusive processes through $g$. On the other hand, if the density fluctuations are out of phase with the temperature fluctuations, the opposite happens.

As we shall see in detail in Sect. 3 the physical origin of thermal diffusion and the sign of its effect (i.e. the sign of the $g$ term) lies with the creation of a particle flux which is parallel to the heat flow itself (that is, antiparallel to the temperature gradient). To determine how this comes about we have to remember that the parameter $g$ is proportional to the derivative of the collision frequency with respect to temperature. The derivative is positive if the collision frequency increases with energy and negative if it decreases with energy.

The sign of the thermal diffusion effect (and therefore the sign of $g$ ) is important and needs to be discussed for a physical understanding of its role: it depends on microscopic collisional properties. As a guide to understanding this, consider the familiar situation of a hard sphere collision, where the collision frequency increases with energy: the collisional cross section, $\sigma$ is then constant and the product $\sigma g_{r}$, where $g_{r}$ is the relative speed between colliding particles, is increasing linearly with $g_{r}$ (the mean collision frequency therefore increases as the square root of the temperature after averaging over a large number of collisions). Another familiar plasma situation is that of a fully ionized plasma, for which the cross section decreases so quickly with $g_{r}$ that the collision frequency actually decreases with the kinetic energy of a particle. This situation makes the most energetic particles in a fully ionized plasma 'run away' by having very few collisions when, for example, an electric field is imposed on the system. In this case the collision cross section goes down quickly with energy because of the 'softness' of the potential, namely, its $1 / r$ behavior. It can in fact be shown that for a central force in $1 / r^{4}$ (as is found for the polarization interaction between an ion and a neutral), the collision frequency only goes down inversely proportional to the relative speed of the colliding particles so that the collision frequency in that case is constant (e.g., Hirschfelder et al., 1954). As a result, for Coulomb collisions $g$ is negative, while for 'Maxwell molecule interactions' $\left(1 / r^{4}\right.$ potential) $g$ is simply zero, and for a hard-sphere interaction, $g$ turns out to be $1 / 2$, independently of $T_{e 0}$. For the Schunk and Nagy (1978) formulas that we have adopted in our work and use later (e.g., Kissack et al., 1995), the value turns out to be approximately $5 / 6$, and to vary slightly with temperature. There is a wide 
consensus on using this value for electron-neutral collisions. For instance, Shalimov and Haldoupis (1995) also used $5 / 6$.

This discussion is meant to clarify that, depending on the interaction potential, the particle flow induced by thermal diffusion can, through $g$, either be parallel or antiparallel to the temperature gradient. For magnetized electrons interacting with neutrals, it will be antiparallel. This result can be understood even without looking at equations. To that goal, however, we need the cartoon drawn in Fig. 2. The cartoon illustrates what happens when a hot collisional region is sitting side by side with a cold, and, in this case, much less collisional region. There is a strong magnetic field imposed on the electrons, so that in the absence of an electric field they normally simply stay put and gyrate locally. However, the arrows indicate that after a collision the electrons are scattered in random directions. At the moment they collide they can therefore move a little along the directions shown by the arrows. The density of arrows indicate the number of collisions occurring in each region. It simply illustrates that there are in this case more collisions in the hot region. This effect produces no net flux of electrons except in the gradient region, where for the assumed collision model, more electrons are scattered toward the low-temperature region than toward the high-temperature region since there are more collisions in the hot region. Therefore, if we forget about all other processes that can produce fluxes (using the principle of superposition for the linearized system in order to isolate our particular process), we end up with a flow in the direction opposite to the temperature gradient if $g>0$. The effect changes sign if the collision frequency is greater in the colder region, namely, if $g<0$. In other words the particle flux driven by the heat flow is in the $-g \nabla T_{e}$ direction, namely, in the same direction as the heat flow itself if $g>0$, as in the $E$ region electron case dealt with here.

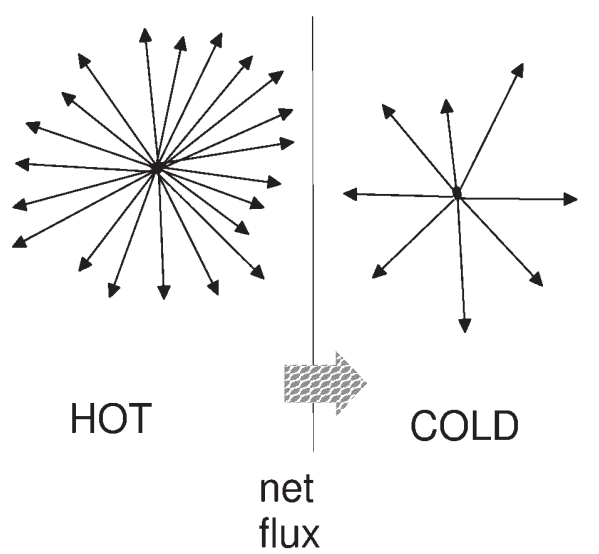

Fig. 2. Cartoon representing electron scattering at one point from a hot region and one point from a cold region. The collision frequencies are assumed to be larger in the hot region. A net flux of particles is produced at the interface owing to the difference in collision frequencies

\subsection{How thermal instabilities work}

The cartoon in Fig. 3 might help sort out further what is happening when we put together the thermal mechanisms that contribute to destabilize the plasma, namely, (1) some mechanism (like friction in the Pedersen conductivity instability case) that creates anticorrelations between density and temperature fluctuations and (2) thermal diffusion, which amplifies these anticorrelations. In the cartoon of Fig. 3 we show the oscillations undergone by three parameters involved in the wave dynamics in an unstable Farley-Buneman situation aided by those positive thermal feedbacks. The top wave form is for the perturbed density $\delta n_{e} / n_{0}(N)$, the middle one for the perturbed temperature $\delta T / T_{e 0}(T)$, and the bottom one for the perturbed pressure $\delta p_{e} / p_{e} 0$ $(P)$. Fluxes in the top wave move the plasma from regions of low density to regions of high density: this is the Farley-Buneman mechanism (FB), and is a result of a positive feedback from ion inertia and perturbed electric fields (see Sect. 2.1 or Fejer et al., 1984, for a more thorough discussion). Below the density wave, we show the temperature wave taking plasma from a hot region to a cold region through the thermal diffusion process (TD) described in the previous subsection; this corresponds to the $g$ positive situation, namely, the one most likely to exist in the $E$ region, and the one that amplifies thermal instability. Note that ion inertia is not involved in thermal diffusion, a fact that will become important when we discuss the simultaneous presence of thermal diffusion and Farley-Buneman effects. This takes us to the pressure wave $(P)$, where we show that the role played by ordinary diffusion (OD) is to try to remove the pressure fluctuations. This last process operates through the terms in $C_{s}^{2} \nabla^{2} \delta n$ and the term 1 in $(1+g)$ in the temperature equivalent, $C_{s}^{2} \nabla^{2} \delta T_{e}$; it tries to remove pressure structures at a rate proportional to the ion speed of sound in the medium (note that the pressure fluctuations are weakened when $\delta T_{e}$ is anticor-

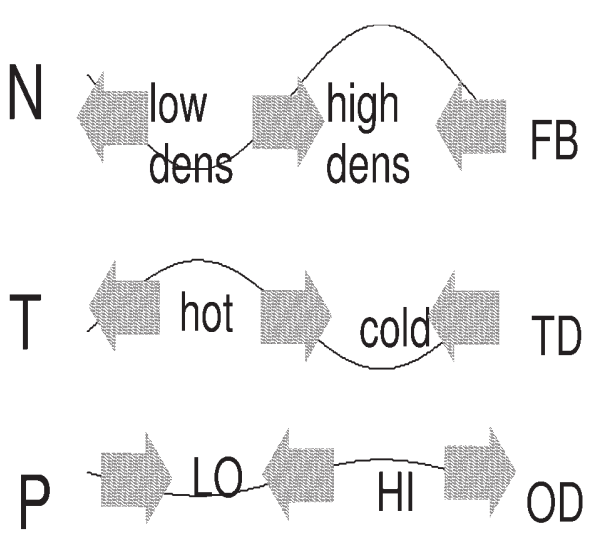

Fig. 3. Diagram showing the particle fluxes produced by the FarleyBuneman process in the density wave (top), by thermal diffusion in the temperature wave (middle) and by ordinary diffusion in the pressure wave (bottom). This sketch is valid for hard-sphere types of elastic collisions and zero aspect angles 
related to $\delta n$ as in the case presented in the cartoon). The presence of the pressure gradient effect is, of course, the reason for requiring the electron drift to exceed the ionacoustic speed in normal isothermal Farley-Buneman situations. Our cartoon should help clarify that the reason a system might trip into a thermal instability is not that the ion-acoustic speed goes to zero (or even becomes imaginary), but rather that the pressure fluctuations are becoming small while the FarleyBuneman feedback mechanism and the thermal diffusion mechanisms are simultaneously enhancing the density fluctuations while reducing the total pressure fluctuations, thus allowing waves to grow more easily.

\section{More detailed mathematical treatment of thermal feedback in the Farley-Buneman instability}

We mentioned in the previous section that at high electron temperatures the treatment of dominant thermal feedback terms is far more difficult to handle when the wave vector is along the $\mathbf{E}_{0} \times \mathbf{B}$ direction as opposed to when it is oriented at the intermediate directions for which the Pedersen conductivity instability is taking place. The aim of this section is to go through the main lines of the thermal feedback derivation along the $\mathbf{E}_{0} \times \mathbf{B}$ direction, and when necessary, to highlight differences between this work and the earlier work presented, in particular, by Shalimov and Haldoupis (1995).

To keep the work more tractable and stay as close to the physics as possible, we do not offer here a general treatment. This means that we stay with the small $\psi$, zero aspect angle, zero flow angle case considered by Gurevich and Karashtin (1984) and Shalimov and Haldoupis (1995). The more general treatment that includes all possible $\psi$ values, aspect and flow angles as well as electron heating by electric fields and plasma waves already exists (Kissack et al., 1995, 1997), but its ramifications can only be understood by exploring particular cases and in the process by comparing the results with the work of others, as is being done here.

As we already indicated in Sect. 2, the electron energy balance can be written in the form

$$
\begin{aligned}
\frac{3}{2} \frac{D}{D t} & \left\{\ln \left(\frac{p_{e}}{n_{e}^{5 / 3}}\right)\right\}=\frac{3}{2} n \frac{D T_{e}}{D t}-T_{e} \frac{D n}{D t} \\
& =-\nabla \cdot \mathbf{q}_{e}-n_{e} \eta\left(T_{e}-T_{n}\right)+n_{e} \epsilon v_{e} m_{e}\left|\mathbf{v}_{e}\right|^{2}
\end{aligned}
$$

where the convective derivative is $D / D t=\partial / \partial t+\mathbf{v}_{e} \cdot \nabla$ and we recall that we have adopted Robinson's (1998) notation for the cooling rate and his $\epsilon$ factor. For the time being the only important points are that $\epsilon$ and $\eta$ will not be considered to be functions of $n_{e}$ or $T_{e}$ and that $\epsilon$ is not more than 10 .

We now consider the perturbed energy equation for a wave vector along the $\mathbf{E}_{0} \times \mathbf{B}$ drift, that is, along the electron zeroth order drift. Since the perturbed drift is then to leading order perpendicular to the zeroth order drift, the heating rate perturbations will not contribute the $\mathbf{V}_{e 0} \cdot \delta \mathbf{v}_{e}$ term that we met in the Pedersen conduc- tivity instability. However, the term $\epsilon v_{e}$ is itself temperature dependent. We will assume here, just for simplicity, that this dependence is felt mostly through $v_{e}$ (the $\epsilon$ factor hides a lot of physics that goes well beyond the scope of the present study). With this in mind, for our particular geometry, the resulting perturbed energy balance is given by

$$
\begin{aligned}
- & i\left(\omega-\mathbf{k} \cdot \mathbf{V}_{e 0}\right)\left[\frac{3}{2} t_{e 1}-n_{1}\right]+i \mathbf{k} \cdot \mathbf{q}_{e 1} \\
& =-\eta t_{e 1}+g \epsilon v_{e} M^{2} t_{e 1}
\end{aligned}
$$

where $M$ is the electron Mach number $\mathbf{V}_{e 0} / \sqrt{T_{e 0} / m_{e}}$ and we are from now on using the symbols $t_{e 1}=\delta T_{e} / T_{e 0}$, $n_{1}=\delta n / n_{0}$, and $\mathbf{q}_{e 1}=\delta \mathbf{q}_{e} / p_{e 0}$. To get result 22, $v_{e}$ has also been expanded into $v_{e}+\delta T_{e} \partial v_{e} / \partial T_{e}$ evaluated at $T_{e 0}$ and the subscript 0 has been dropped from $v_{e 0}$. Also a $T_{e}^{g}$ dependence was assumed for the electron collision frequency. It is easy to show that for a zeroth order energy balance between electron friction and cooling to the neutrals, we can rearrange the last two terms into $\bar{\eta} t_{e 1}=\eta\left(1-g+g T_{n} / T_{e 0}\right) t_{e 1}$. This expression shows that the Mach number could be more than just a simple correction to $\eta$ in the event that $g$ is of order 1 and $T_{e 0}$ is becoming large compared to $T_{n}$. We will therefore use $\bar{\eta}$ instead of $\eta$ from now on.

Next, we have to consider the heat-flow equation. In its full glory it is given by (e.g. Schunk, 1977)

$$
\begin{aligned}
\frac{D_{e} \mathbf{q}_{e}}{D t} & +\frac{7}{5} \mathbf{q}_{e} \cdot \nabla \mathbf{v}_{e}+\frac{7}{5} \mathbf{q}_{e}\left(\nabla \cdot \mathbf{v}_{e}\right)+\frac{2}{5}\left(\nabla \mathbf{v}_{e}\right) \cdot \mathbf{q}_{e} \\
& +\frac{5}{2} \frac{p_{e}}{m_{e}} \nabla T_{e}+\frac{e}{m_{e}} \mathbf{q}_{e} \times \mathbf{B} \\
= & -v_{e}\left(1+\frac{2}{5} g[g+1]\right) \mathbf{q}_{e}-g v_{e} p_{e} \mathbf{v}_{e}
\end{aligned}
$$

At the perturbed level we drop the first four terms using the assumption that the zeroth order heat flows are small and the zeroth order velocities are free of gradients or divergences. The first term is dropped because of the quick response time of the heat flow to the other forces, much like the $D_{e} / D t$ term is always dropped from the perturbed momentum equation in the treatment of low frequency waves. This leaves us with the balance

$$
\begin{aligned}
v_{e}( & \left.1+\frac{2}{5} g[g+1]\right) \mathbf{q}_{e 1}+\Omega_{e} \mathbf{q}_{e 1} \times \hat{\mathbf{b}} \\
= & {\left[-i \frac{5}{2} \mathbf{k} \frac{T_{e 0}}{m_{e}}-g^{2} v_{e} \mathbf{V}_{e 0 \perp}\right] t_{e 1}-g v_{e} \delta \mathbf{v}_{e} }
\end{aligned}
$$

where $\hat{\mathbf{b}}$ is a unit vector in the geomagnetic field direction. Note that things like the term in $g^{2}$ comes from taking appropriate derivatives of the collision frequency with respect to temperature, assuming it behaves as a $T_{e}^{g}$ function to first order.

The momentum equation is now needed if we want to close the system. Suffice it to say that, as with the classical treatment, in the 8-moment description that we are using the contribution of the convective derivative term is dropped from the perturbed momentum equation. The perturbed equation then takes the form 
$v_{e} \delta \mathbf{v}_{e}+\Omega_{e} \delta \mathbf{v}_{e} \times \hat{\mathbf{b}}=\mathbf{S}$

where

$$
\begin{aligned}
\mathbf{S}= & -i \frac{T_{e 0}}{m_{e}} \mathbf{k} n_{1}+\left\{-i \frac{T_{e 0}}{m_{e}} \mathbf{k}-v_{e} g \mathbf{V}_{e 0 \perp}\right\} t_{e 1} \\
& +i \frac{T_{e 0}}{m_{e}} \mathbf{k} \phi_{1}-\frac{2}{5} g v_{e} \mathbf{q}_{e 1}
\end{aligned}
$$

where we have defined $\phi_{1}=e \delta \phi / T_{e 0}$. To leading order in $v_{e} / \Omega_{e}$, the solution to this equation is

$\delta \mathbf{v}_{e}=\frac{v_{e}}{\Omega_{e}^{2}} \mathbf{S}-\frac{\mathbf{S} \times \hat{\mathbf{b}}}{\Omega_{e}}$.

From this it is easy to show that to leading order in $v_{e} / \Omega_{e}$ we get for the divergence of the perturbed flows at zero aspect angle

$\mathbf{k} \cdot \delta \mathbf{v}_{e}=-i k^{2} D_{e}\left\{n_{1}+t_{e 1}-\phi_{1}\right\}-\frac{2 v_{e} g}{5 \Omega_{e}} \mathbf{k} \cdot\left(\mathbf{q}_{e 1} \times \hat{\mathbf{b}}\right)$

We recall that we have used the fact that $\mathbf{k}$ is along the $\mathbf{E}_{0} \times \mathbf{B}$ direction to drop some terms.

We are now in a position to close the system by using our perturbed heat-flow equation in the perturbed momentum equation. To this goal we take advantage of the smallness of $v_{e} / \Omega_{e}$ and obtain a leading order contribution for the heat flow term in the momentum equation. All we have to do is drop all the terms that are proportional to a collision frequency in the perturbed heat flow equation, Eq. (24). This means that for our strongly magnetized electrons we can simply write

$\mathbf{k} \cdot\left(\mathbf{q}_{e 1} \times \hat{\mathbf{b}}\right) \approx-i \frac{5}{2} \frac{k^{2}}{v_{e}} D_{e} t_{e 1}$

Using this simple approximation back into the manipulated momentum balance, Eq. (28), gives us the important result

$\mathbf{k} \cdot \delta \mathbf{v}_{e}=-i k^{2} D_{e}\left\{n_{1}+(1+g) t_{e 1}-\phi_{1}\right\}$.

We note that this result is equivalent to what Shalimov and Haldoupis (1995) obtained in their Eq. (5).

We also need an expression for the divergence of the perturbed heat flow in Eq. (22), before we can go back to the perturbed energy equation. To this goal, using Eq. (24) and a solution of the type given by Eq. (27), we first write to leading order in $v_{e} / \Omega_{e}$

$$
\begin{aligned}
\mathbf{q}_{e 1}= & \frac{v_{b}}{\Omega_{e}^{2}}\left(+i \frac{5}{2} \mathbf{k} \frac{T_{e 0}}{m_{e}} t_{e 1}-g v_{e} \delta \mathbf{v}_{e}\right) \\
& -\frac{1}{\Omega_{e}}\left(+i \frac{5}{2} \mathbf{k} \times \hat{\mathbf{b}} \frac{T_{e 0}}{m_{e}} t_{e 1}-g v_{e} \delta \mathbf{v}_{e} \times \hat{\mathbf{b}}\right)
\end{aligned}
$$

where $v_{b}=v_{e}(1+2 g(g+1) / 5)$. Clearly, for $\mathbf{k} \cdot \mathbf{q}_{e 1}$ the leading order contributions in $v_{e} / \Omega_{e}$ will have to come from the $\delta \mathbf{v}_{e} \times \hat{\mathbf{b}}$ term plus the very first term. This gives us the relatively simple expression

$i \mathbf{k} \cdot \mathbf{q}_{e 1}=k^{2} g D_{e} n_{1}-k^{2} g D_{e} \phi_{1}+k^{2} g_{b} D_{e} t_{e 1}$

where $g_{b}=5 / 2+2 g+g^{2}$ is basically $g$ plus a contribution from thermal conduction.
We can now finally go back to the perturbed energy equation, Eq. (22), and eliminate the heat flow contribution from it using what we have just obtained. The result is

$$
\begin{gathered}
{\left[-\bar{\eta}+i \frac{3}{2}\left(\omega-\mathbf{k} \cdot \mathbf{V}_{e 0}\right)-k^{2} g_{b} D_{e}\right] t_{e 1}} \\
=i \mathbf{k} \cdot \delta \mathbf{v}_{e}+k^{2} g D_{e} n_{1}-k^{2} g D_{e} \phi_{1} \\
=k^{2}(g+1) D_{e}\left(n_{1}+t_{e 1}-\phi_{1}\right) .
\end{gathered}
$$

This can be rearranged slightly to give

$$
\begin{aligned}
& \left\{i \frac{3}{2}\left(\omega-\mathbf{k} \cdot \mathbf{V}_{e 0}\right)-k^{2} D_{e}\left[\left(\frac{5}{2}+g\right)+(g+1)^{2}\right]-\bar{\eta}\right\} t_{e 1} \\
& \quad=i \mathbf{k} \cdot \delta \mathbf{v}_{e}+k^{2} g D_{e} n_{1}-k^{2} g D_{e} \phi_{1} \\
& \quad=k^{2}(g+1) D_{e}\left(n_{1}+t_{e 1}-\phi_{1}\right) .
\end{aligned}
$$

We have used the perturbed electron continuity equation, $\left(\omega-\mathbf{k} \cdot \mathbf{V}_{e 0}\right) n_{1}=\mathbf{k} \cdot \delta \mathbf{v}_{e}$ to obtain the expressions after the second equal sign. To close the system, we use it once again to get from Eq. (30)

$$
\omega-\mathbf{k} \cdot \mathbf{V}_{e 0}+i k^{2} D_{e}\left(1-\frac{\phi_{1}}{n_{1}}\right)+i k^{2} D_{e}(1+g) \frac{t_{e 1}}{n_{1}}=0 .
$$

Finally, the standard treatment of the ion continuity and momentum equations at the perturbed level gives the familiar expression

$1-\frac{\phi_{1}}{n_{1}}=\frac{T_{e 0}+T_{i 0}}{T_{e 0}}\left(1-\frac{\omega^{2}}{k^{2} C_{s}^{2}}-\frac{i \omega v_{i}}{k^{2} C_{s}^{2}}\right)$.

Using the last two equations we get the dispersion relation in a form already presented in the previous section, namely,

$$
\begin{gathered}
\omega-\mathbf{k} \cdot \mathbf{V}_{e 0 \perp}+\frac{\psi}{v_{i}}\left\{i k_{\perp}^{2} \frac{T_{i 0}}{m_{i}}+i k_{\perp}^{2} \frac{T_{e 0}}{m_{i}}\left[1+(1+g) \frac{t_{e 1}}{n_{1}}\right]\right. \\
\left.+\omega\left(v_{i}-i \omega\right)\right\}=0 .
\end{gathered}
$$

To find the solution to the dispersion relation we still need to obtain an explicit expression for $t_{e 1} / n_{1}$. To this goal we can use Eqs. (30) and (33). By isolating a term in $k^{2} D_{e}\left(1-\phi_{1} / n_{1}\right)$ in each of these equations we obtain

$$
\begin{aligned}
& g\left[\frac{i \mathbf{k} \cdot \delta \mathbf{v}_{e}}{n_{1}}-k^{2} D_{e}(1+g) \frac{t_{e 1}}{n_{1}}\right] \\
& \quad=\frac{i \mathbf{k} \cdot \delta \mathbf{v}_{e}}{n_{1}}\left(\frac{3}{2} \frac{t_{e 1}}{n_{1}}-1\right)-\frac{t_{e 1}}{n_{1}}\left(\bar{\eta}+k^{2} g_{b} D_{e}\right) .
\end{aligned}
$$

This gives a complex quadratic equation for $t_{e 1} / n_{1}$, namely,

$$
\left(\frac{t_{e 1}}{n_{1}}\right)^{2}+A \frac{t_{e 1}}{n_{1}}-\frac{2}{3}\left(1-\frac{\phi_{1}}{n_{1}}\right)=0
$$


where

$A=\frac{1-\frac{\phi_{1}}{n_{1}}-\frac{2}{3} X}{1+g}$,

and where

$X=g^{2}+3 g+\frac{7}{2}+\frac{\bar{\eta}}{k^{2} D_{e}}$.

By solving simultaneously for $t_{e 1} / n_{1}$ and the dispersion relation, Eq. (37), we can in principle find the roots of the equation and determine the influence of thermal diffusion on the instability. Note that an entirely equivalent expression for $t_{e 1} / n_{1}$ is also used in the appendix (Eq. 44). Each expression has advantages and inconvenients as far as calculation algorithms are concerned. The important point is that the two different approaches produce the same roots for the complex frequency in the dispersion relation, Eq. (37).

\section{Calculations of thermal feedback effects in the $E_{0} \times B$ direction for heating rates that depend linearly on electron density}

In this section we study the solution to the dispersion relation obtained in the preceding section. We recall that the wave vector is in the $\mathbf{E}_{0} \times \mathbf{B}$ direction, the aspect angle is zero, and the heating rate depends linearly on electron density. Finally, we focus on this situation because it has been suggested that waves a few meters in size should be unstable at speeds less than the isothermal ion-acoustic speed when the electron temperature is high (Shalimov and Haldoupis, 1995). Here we have repeated the latter authors' calculations for several reasons. A first reason is that, while we agree with most of the expressions derived by Shalimov and Haldoupis (1995), there are unfortunately two possibly important differences between their results and ours with regard to Eq. (34) (their Eq. 4). Both differences involve the product $g i k v_{e 0}$ which is simply not present in our work. This term in the Shalimov and Haldoupis paper is of the form $g i k v_{e 0}\left(t_{e 1}+n_{1}\right)$ and can be traced to a contribution from what they call a transverse part of the heat flow contribution. As we have seen, because of the magnetization effects, this term simply does not belong there, since it should have been of order $v_{e}^{2} / \Omega_{e}^{2}$ smaller, had the proper expression been used. It is not clear to us how this oversight affected the results published by Shalimov and Haldoupis (1995).

A second reason for repeating the calculations is that in their numerical calculations, Shalimov and Haldoupis (1995) appear to have neglected ion inertia for a computation of the thermal feedback terms per se. Our formulation is actually more transparent and also more amenable to including all contributions. In other words, it is completely straightforward for us to keep all the terms including ion inertia in the temperature fluctuation term. Indeed, we have checked that ion inertia has a very significant influence on the thermal feedback terms: as will be shown in the Appendix as well as in the next subsection, the full inclusion of ion inertia in thermal feedback effects always has a stabilizing influence on the waves. By contrast, the removal of ion inertial effects $\left(\omega^{2}\right.$ terms) in the calculation of $t_{e 1} / n_{1}$ in Eq. (39) (through the $\phi_{1}$ terms) does yield results that closely resemble those obtained by Shalimov and Haldoupis (1995). That is to say: the exclusion of ion inertia in the treatment of the temperature fluctuation does indeed allow thermal diffusion to destabilize the plasma waves and leads to erroneous physical conclusions.

A final reason for repeating the Shalimov and Haldoupis (1995) calculations is that we have noted that it is important to replace the original cooling coefficient $\eta$ by $\bar{\eta}$, which is significantly reduced from $\eta$ owing to canceling contributions from heating rates when thermal diffusion is important.

\subsection{Linear eigenfrequencies and growth rates}

The thermal feedback terms introduced in the previous section affect both the eigenfrequency and the growth rate of the Farley-Buneman instability. A representative set of solutions is shown in Figs. 4 and 5. For these figures we selected a set of geophysical parameters appropriate for $110 \mathrm{~km}$ altitude and introduced a $2000 \mathrm{~m} / \mathrm{s}$ electron drift, $V_{e 0}$. We studied the behavior of the phase velocity and growth rate as functions of wavelength and electron temperature. The electron drift was taken as large, to be consistent with the fact that we are interested here mostly in hot electron temperature events, which are known to take place only when the $\mathbf{E}_{0} \times \mathbf{B}$ drift is large. This being said we do not consider a particular connection between the electron tempera-

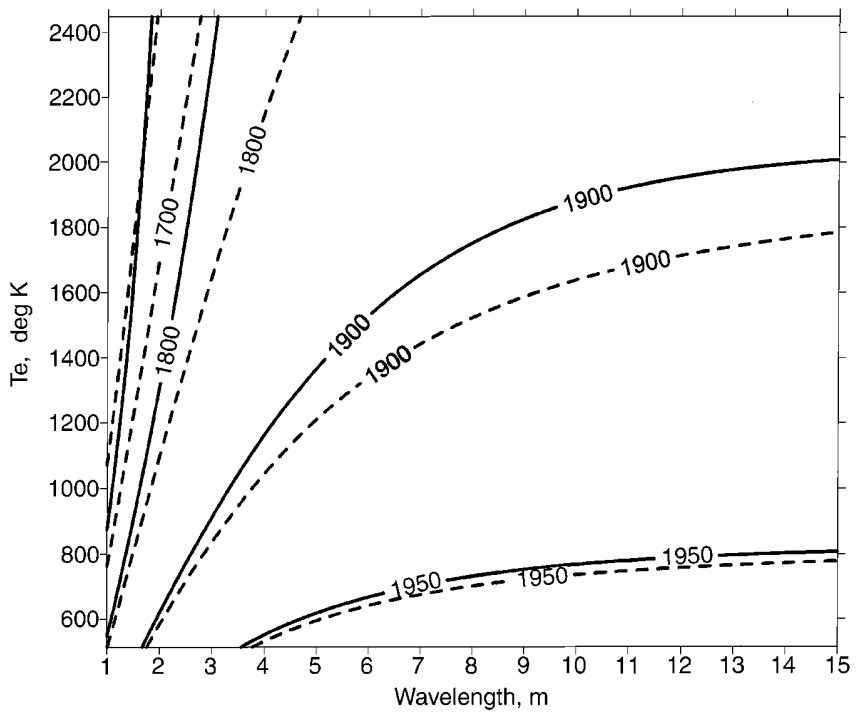

Fig. 4. Contours of the phase velocities $(\mathrm{m} / \mathrm{s})$ of the Farley-Buneman instability for wave vectors parallel to the $\mathbf{E}_{0} \times \mathbf{B}$ drift. The calculations were made for a $2000 \mathrm{~m} / \mathrm{s}$ electron drift at $110 \mathrm{~km}$ altitude. Solid contour lines: exact solutions to the classical isothermal $\left(t_{e 1}=0\right)$ Farley-Buneman instability. Dashed contour lines: exact solutions to the Farley-Buneman instability using Eqs. (37) and (39) 


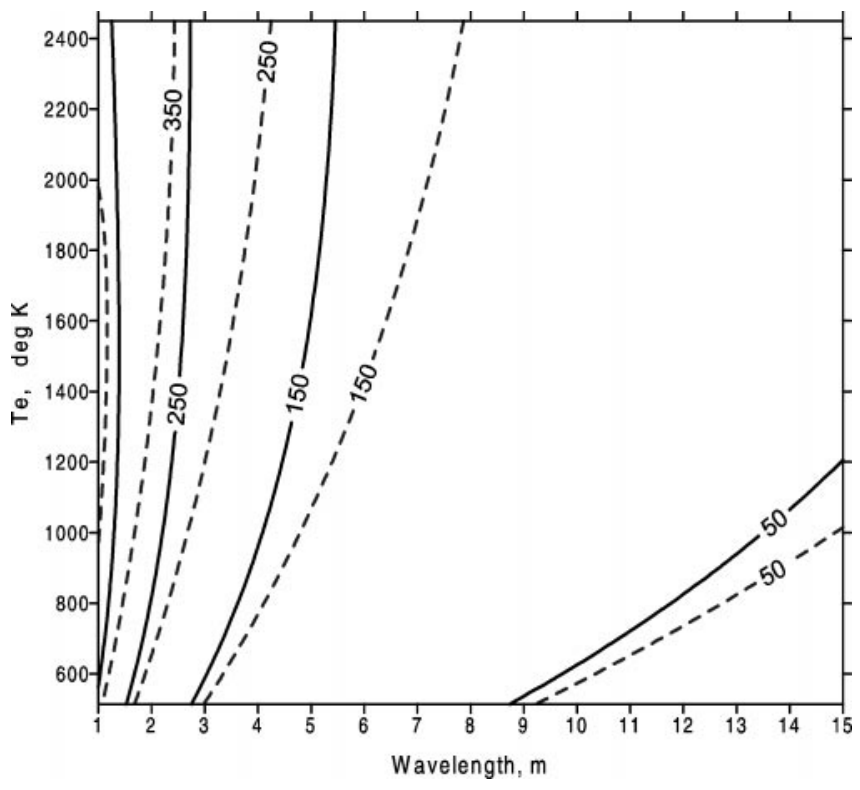

Fig. 5. Same as in Fig. 4, but for the growth rate divided by the wave number, in $\mathrm{m} / \mathrm{s}$

ture and the electron drift at this point: the selection of a particular model of the relation between $T_{e}$ and $V_{e 0}$ is not relevant for what we are about to discuss and will be kept for the next subsection.

The most important point about Figs. 4 and 5 is that, as far as wave vectors aligned with the direction of the $\mathbf{E}_{0} \times \mathbf{B}$ drift are concerned, both the phase velocities and the growth rates are smaller at a given $\left(T_{e}, \lambda\right)$ point when we introduce thermal feedback terms (including thermal diffusion) than when we neglect them in the treatment of the Farley-Buneman instability. Note that the solutions posted in Figs. 4 and 5 are the exact solutions to Eqs. (37) and (39) (or, equivalently, Eq. (44) in the Appendix, in lieu of Eq. (39)). Clearly, the fact that the phase velocity lines tend to be a strong function of wavelength at small wavelengths means that the approximate solution given by Eq. (3) does not work that well at a few meters and less, even for the classic isothermal Farley-Buneman mechanism. The reason is that, at small wavelengths, the growth rate becomes large enough to affect the real part of the frequency by creating a measurable decrease in the approximation for the phase velocity that we have obtained in Eq. (3). When thermal feedback effects are added that feature remains. In fact, one other point about Fig. (4) is that the phase velocity is not affected very much by thermal feedback effects (about 5\% or less in Fig. 4). The growth rates (Fig. 5) are more strongly affected if we think in terms of relative differences.

Figures 4 and 5 therefore indicate that thermal feedback effects have a relatively strong stabilizing influence on the solution. This is confirmed analytically when we study very small growth rate solutions (even though, strictly speaking, the requirement of large drifts during electron heating effects precludes having such a condition physically). We show in the Appendix that for small positive growth rates the thermal feedback terms always lead to a decrease in the rates, consistent with what we have found in Fig. 5 .

\subsection{Extrapolation to the nonlinear regime}

Experience shows, not surprisingly, that the linear theory of fast growing waves is not useful in describing the nonlinear wave properties that make up radar and rocket observations. In other words, under strong destabilizing conditions, the mean phase velocity of the waves observed by radars or rockets does not match the value given by Eq. (3) or obtained in Fig. 4 (see for example the comparison obtained by Nielsen and Schlegel, 1983, between EISCAT and STARE data over a common plasma volume). Instead, the observed mean phase velocity is comparable to that which is obtained over zero growth rate conditions.

An intuitive way to understand the detection of threshold speeds at large amplitudes is to realize that radar observations are biased to the largest amplitude waves, at a point where their instantaneous growth rate goes from positive to negative. Observing the large amplitude waves with a Doppler shift corresponding to threshold drift velocities is therefore not so surprising. On the more quantitative side, several theoretical ideas have been formulated to determine just how this may be happening for wave vectors aligned with the $\mathbf{E}_{0} \times \mathbf{B}$ direction (primary waves). In particular Sudan (1983) proposed the notion of anomalous diffusion which was later utilized by Robinson (1986). According to this approach the microturbulence associated with the growth of a particular wave creates an increase in electron scattering that only stops when the scattering is intense enough to stop the growth of the unstable waves. Hamza and St.-Maurice (1993) proposed modecoupling as an alternative mechanism. According to that scheme, the energy of a mode is given off to other modes so that on average the growth rate ends up being zero while the spectrum broadens.

Irrespective of one's particular choice of a wave saturation mechanism, an empirical notion that is widely used in the field is that the large amplitude waves move at the threshold speed associated with the particular instability mechanism responsible for their growth. Shalimov and Haldoupis (1995) themselves have used this notion in their Sect. 3 when they looked at the threshold conditions of their thermal diffusion mechanism to infer that electron heating effects were responsible for the observation of slow moving type III waves. Using that same approach, we have also sought to determine how threshold conditions are affected by thermal processes, using our own theoretical results (see Eq. (52) in the Appendix). Thus, in spite of the fact that the magnitude of the $\mathbf{E}_{0} \times \mathbf{B}$ drift is well above the Farley-Buneman threshold speed when the electrons are heated, we have computed the frequencies (or phase velocities) required to get zero growth rates. We have taken the position that the nonlinear eigenfrequency shift comes from processes such as mode-coupling or 
anomalous diffusion and is of no concern to us here. Our bottom line is the existence a nonlinear shift in frequency yielding a nonlinear null growth rate under phase velocity conditions that closely resemble the linear null growth rate conditions.

In Fig. 6 we present zero growth rate conditions obtained by solving Eq. (52) for $\Gamma=0$ for $3 \mathrm{~m}$ waves at $110 \mathrm{~km}$. We selected $3 \mathrm{~m}$ waves in Fig. 6 because many radar measurements are made around $50 \mathrm{MHz}$, which corresponds to this wavelength regime. Other wavelengths yield similar numbers. Note also that by contrast to Figs. 4 and 5 we are now providing a rough guide for the range of observed electron temperatures as a function of electron drift as well. This region is indicated by the area between the two light lines of increasing electron temperature with electron drift speed in Fig. 6. The area thus covered is based on the 'normal' data recently presented by St.-Maurice et al. (1999) and is also in basic agreement with the numbers given by Robinson (1986).

In Fig. 6 the isothermal ion-acoustic speed is shown by the strictly vertical solid contour lines. Recall that these are the threshold speed values if we ignore thermal feedback effects. The dashed contour lines are the threshold speeds in the presence of thermal feedback. We notice that, consistent with the results inferred from Figs. 4 and 5, the threshold speeds are considerably higher than the threshold speeds given by the isothermal ion-acoustic speed (in addition, once a high enough electron temperature is reached, the threshold speed becomes a function of electron drift only. This behavior

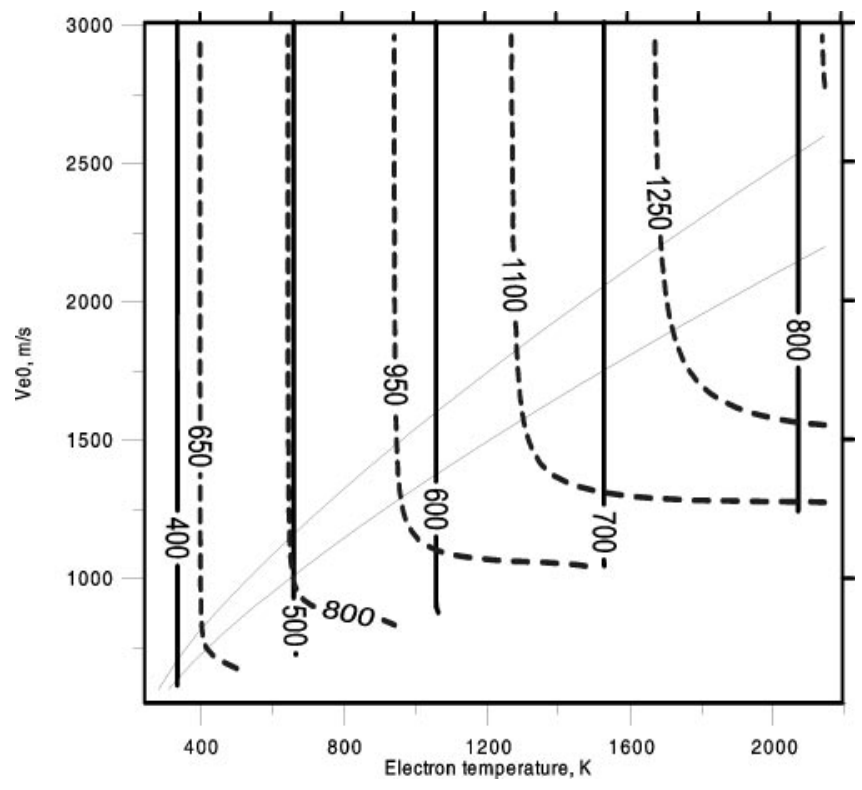

Fig. 6. Contours of the threshold speed $(\mathrm{m} / \mathrm{s})$ of $3 \mathrm{~m}$ FarleyBuneman waves at $110 \mathrm{~km}$. We show only the regions for which the electron drift exceeds the threshold speed by a sufficient amount. Solid contours: classic isothermal Farley-Buneman waves. Dashed contours: Farley-Buneman waves with thermal feedback when the wave vector is parallel to the $\mathbf{E}_{0} \times \mathbf{B}$ drift. The two light traces, which are only meant as a guide for the reader, approximate the lower bound and upper bound normally observed in the electron temperature near $110 \mathrm{~km}$, based on the work of St.-Maurice et al., (1999) is more pronounced at shorter wavelengths but is not shown here).

An important feature of Fig. 6 is that the inferred threshold phase speeds are very reminiscent of the behavior of 'type IV' waves. These waves are observed in the presence of strong electron heating (Farley and Providakes, 1989) and are characterized by mean Doppler shifts of the order of $1000 \mathrm{~m} / \mathrm{s}$, that is, by speeds that are well above the values expected for the isothermal ionacoustic speed. Indeed, Farley and Providakes (1989) found the waves from the observations to be along the $\mathbf{E}_{0} \times \mathbf{B}$ direction (in agreement with the fact that this has to be the direction for which the threshold speeds are highest, according to our presentation), and the phase speeds to be substantially larger than the isothermal ionacoustic speed, just as we have found here. Specifically, at $112 \mathrm{~km}$, in the example treated by Farley and Providakes (1989), $T_{e}$ was about $1400 \mathrm{~K}$ while the phase speed of the type IV waves was about $1100 \mathrm{~m} / \mathrm{s}$. This compares quite favorably with our inference from Fig. 6, which would put the threshold phase speed in the range 1100 to $1150 \mathrm{~m} / \mathrm{s}$ at $110 \mathrm{~km} / \mathrm{s}$ (and very similar speeds at nearby altitudes). As Fig. 6 also shows, the isothermal ion-acoustic is only about $675 \mathrm{~m} / \mathrm{s}$ for the same conditions. Our work, in combination with the Farley and Providakes (1989) results therefore strongly suggests that the type IV waves observed at $50 \mathrm{MHz}$ owe their properties to thermal feedback effects.

We should finally note that there is little wavelength dependence in the threshold speed for the parameters used in Fig. 6. The threshold speed tends to decrease somewhat towards smaller wavelength. But even that decrease is not significant, nor does it mean a lot, since a kinetic theory should really be used to obtain accurate results at these smaller wavelengths. We should perhaps note that there is a more pronounced wavelength dependence at higher temperatures when we go down in altitude (not shown here), but the effect is limited to smaller wavelengths and is only felt at rather high electron temperatures.

In conclusion, the introduction of thermal feedback effects in the treatment of Farley-Buneman waves has a strong stabilizing influence on the instability when the wave vector is aligned with the $\mathbf{E}_{0} \times \mathbf{B}$ drift vector. As a consequence, the threshold speed goes up when the waves reach their nonlinear saturation amplitude. Contrary to Shalimov and Haldoupis (1995) we therefore conclude that thermal feedback might be responsible for the observation of fast moving type IV waves rather than for the excitation of slowly moving type III waves. Our conclusion that thermal feedback has a stabilizing influence is based on the same physics as Shalimov and Haldoupis (1995), but it relies on a full solution to dispersion relation (in addition to introducing a couple of algebraic corrections to the earlier work).

\section{Summary and conclusions}

We have studied how thermal feedback terms modify the Farley-Buneman instability at zero aspect angle in the 
presence of $E$ region electron heating at high latitudes. We have shown that thermal effects can stimulate the growth of Farley-Buneman waves if and only if the temperature fluctuations are 'out of phase' with the density fluctuations (more precisely, if and only if the phase difference between temperature fluctuations and density fluctuations is between $\pi / 2$ and $3 \pi / 2$ with a phase lag of $\pi$ for the largest effects). We have discussed a situation for which this thermal destabilization takes place, namely, the Pedersen conductivity instability, when velocity perturbations can create negative temperature fluctuations in regions of positive density fluctuations. This anticorrelation is triggered by a reduction in the frictional heating rate caused by velocity fluctuations of a sign such as to decrease the instantaneous drift of the electron gas.

We have determined that thermal diffusion cannot change the sign of the waves growth rate at zero aspect angle. However, thermal diffusion amplifies thermal feedback effects. That is to say, if the plasma is destabilized by thermal effects, as in the Pedersen instability case, thermal diffusion makes the plasma even more unstable. Vice-versa, when the wave vector is parallel to the $\mathbf{E}_{0} \times \mathbf{B}$ drift direction, thermal effects make the plasma less unstable, and thermal diffusion amplifies the effect. We have shown that the thermal diffusion amplification effect is due to a particle flux that moves the electrons against the temperature gradient when the magnetized electrons collide with the neutral gas.

Finally, we have argued that with wave vectors parallel to $\mathbf{E}_{0} \times \mathbf{B}$ thermal effects lead to higher threshold speeds. We have suggested that this may be related to the occurrence of the 'type IV' waves observed with $50 \mathrm{MHz}$ radars, with phase speeds of the order of $1000 \mathrm{~m} / \mathrm{s}$. It follows from this kind of reasoning that the opposite should take also place when thermal effects help destabilize the plasma. Specifically, one can see from the Pedersen instability threshold condition, Eq. (17), that when the wave vector is at about $45^{\circ}$ to the $\mathbf{E}_{0} \times \mathbf{B}$ direction the lowered thresholds could become important enough to be at the origin of 'type III' waves, namely: the type III waves would be 'primary' waves (we assume here that waves with narrower spectra do not owe their existence to mode coupling) that have a mean Doppler shift at saturation that is markedly less than the ion acoustic speed. As a case in point for the $110 \mathrm{~km}$ altitude parameters that we have used, even with $\epsilon=1$ in Eq. (17), at $\theta=45^{\circ}$ we find that electron drifts of the order of $3 C_{s}$ or more will reduce the threshold speed to $0.7 C_{s}$ and possibly much less. In summary, thermal feedback effects associated with elevated electron temperatures might be responsible for the observation of both type III and type IV wave spectra at high latitudes.

Acknowledgements. We are very grateful to both referees for their very useful insights and comments. In large part because of their comments this work has evolved considerably from its original version. This work was supported by a research grant from the Natural Sciences and Engineering Research Council of Canada. JPSM also thanks the French CNRS for its support while doing some of this research. The Editor in chief thanks two referees for their help in evaluating this paper.

\section{Appendix A: formal proof that thermal diffusion cannot destabilize the E-region plasma when $k \| E_{0} \times B$}

In the main body of the study, we have indicated that thermal diffusion cannot, by itself, destabilize the plasma. In this appendix we present the formal proof of this statement by giving an alternative calculation of $t_{e 1} / n_{1}$ and of the linear growth rate.

As with our earlier discussion, we consider, the situation where $\mathbf{k} \| \mathbf{E}_{0} \times \mathbf{B}$ ( $0^{\circ}$ flow and aspect angles), where the Pedersen conductivity instability mechanism essentially does not contribute. We also assume that the heating term in the energy equation is of the Robinson (1998) form, as used in Eq. (21). That is, it is proportional to $n_{e}$ and has the form $n_{e} \epsilon v_{e} m_{e}\left|\mathbf{v}_{e}\right|^{2}$, with $\epsilon$ being a constant. As mentioned earlier, for traditional Joule heating, $\epsilon=1$.

We have seen in Eq. (35) that we get from the electron continuity equation

$\omega-\mathbf{k} \cdot \mathbf{V}_{e 0}+i k_{\perp}^{2} D_{e}\left\{\left(1-\frac{\phi_{1}}{n_{1}}\right)+(1+g) \frac{t_{e 1}}{n_{1}}\right\}=0$

while using the expression for $i \mathbf{k} \cdot \mathbf{q}_{e 1}$ from Eq. (32) in the linear energy Eq. (22) gives

$$
\begin{aligned}
(\omega & \left.-\mathbf{k} \cdot \mathbf{V}_{e 0}\right)\left[\frac{3}{3} \frac{t_{e 1}}{n_{1}}-1\right] \\
& +i k_{\perp}^{2} D_{e}\left[g\left(1-\frac{\phi_{1}}{n_{1}}\right)+g_{b} \frac{t_{e 1}}{n_{1}}\right]=i \bar{\eta} \frac{t_{e 1}}{n_{1}}
\end{aligned}
$$

where $\bar{\eta}=\eta-g \epsilon M^{2} v_{e}$ and $M^{2}$ is the square of the electron Mach number given by $M^{2}=\left|\mathbf{V}_{e 0}\right|^{2} /\left(T_{e 0} / m_{e}\right)$. Eliminating $1-\left(\phi_{1} / n_{1}\right)$ from these equations gives an expression for $t_{e 1} / n_{1}$

$\frac{t_{e 1}}{n_{1}}=\frac{-i(2 / 3)(1+g)\left(\omega-\mathbf{k} \cdot \mathbf{V}_{e 0}\right)}{(2 / 3) \bar{\eta}-i\left(\omega-\mathbf{k} \cdot \mathbf{V}_{e 0}\right)+k_{\perp}^{2} D_{e}(5+2 g) / 3}$

where we have used the fact that

$\frac{2}{3}\left[g_{b}-g(1+g)\right]=\frac{5+2 g}{3}$

which follows from the expression for $g_{b}$ just after Eq. (32).

Note that since $g \sim O(1)$, we see from Eq. (44) that both the modulus as well as the real part of $t_{e 1} / n_{1}$ is at most of order unity. Thus the size of the relative temperature perturbations $\delta T_{e} / T_{e 0}$ cannot greatly exceed that of the relative density perturbations, $\delta n / n_{0}$.

As we will need the real part of $t_{e 1} / n_{1}$ in calculating the linear growth rate is is useful to multiply the numerator and denominator of Eq. (44) by the complex conjugate of the denominator. Doing this gives

$$
\begin{aligned}
\frac{t_{e 1}}{n_{1}}= & \left\{(2 / 3)(1+g)\left|\omega-\mathbf{k} \cdot \mathbf{V}_{e 0}\right|^{2}-i(2 / 3)(1+g)\right. \\
& \left.\times\left(\omega-\mathbf{k} \cdot \mathbf{V}_{e 0}\right)\left[(2 / 3) \bar{\eta}+k_{\perp}^{2} D_{e}(5+2 g) / 3\right]\right\} \\
& /\left\{\left[(2 / 3) \bar{\eta}+k_{\perp}^{2}(2 / 3) D_{e}(5+2 g) / 3\right]^{2}+\left|\omega-\mathbf{k} \cdot \mathbf{V}_{e 0}\right|^{2}\right\} .
\end{aligned}
$$


Let us write the complex angular frequency of the wave as $\omega=\omega_{r}+i \Gamma$, where $\Gamma$ is the linear growth rate of the waves. Near threshold, with $|\Gamma| \ll\left|\omega_{r}\right|$ and keeping only linear terms in $\Gamma$, we obtain the real part of $t_{e 1} / n_{1}$ from Eq. (46):

$$
\begin{aligned}
& \operatorname{Re}\left(\frac{t_{e 1}}{n_{1}}\right) \\
& =\xi \Gamma+\frac{(2 / 3)(1+g)\left(\omega_{r}-\mathbf{k} \cdot \mathbf{V}_{e 0}\right)^{2}}{\left[(2 / 3) \bar{\eta}+k_{\perp}^{2}(2 / 3) D_{e}(5+2 g) / 3\right]^{2}+\left(\omega_{r}-\mathbf{k} \cdot \mathbf{V}_{e 0}\right)^{2}}
\end{aligned}
$$

where

$$
\xi=\frac{(2 / 3)(1+g)\left[(2 / 3) \bar{\eta}+k_{\perp}^{2} D_{e}(5+2 g) / 3\right]}{\left[(2 / 3) \bar{\eta}+k_{\perp}^{2}(2 / 3) D_{e}(5+2 g) / 3\right]^{2}+\left(\omega_{r}-\mathbf{k} \cdot \mathbf{V}_{e 0}\right)^{2}} .
$$

The appearance of Eq. (47) shows that for this case, at exact threshold conditions, $\operatorname{Re}\left(t_{e 1} / n_{1}\right)$ is positive definite, provided $g>-1$. From the discussion in Sect. 2.4, this is almost certainly true, and consequently the real parts of $\delta T_{e}$ and $\delta n$ are in phase.

Recall from Eq. (37) that the dispersion relation can be written in the form

$$
\begin{gathered}
\omega-\mathbf{k} \cdot \mathbf{V}_{e 0} \\
+\frac{\psi}{v_{i}}\left\{i k_{\perp}^{2} \frac{T_{i 0}}{m_{i}}+i k_{\perp}^{2} \frac{T_{e 0}}{m_{i}}\left[1+(1+g) \frac{t_{e 1}}{n_{1}}\right]\right. \\
\left.+\omega\left(v_{i}-i \omega\right)\right\}=0 .
\end{gathered}
$$

By regrouping the $\omega v_{i}$ term with the electron Doppler term and then dividing by $(1+\psi)$, we can recast the preceding equation as

$$
\begin{gathered}
\omega-\frac{\mathbf{k} \cdot \mathbf{V}_{e 0}}{1+\psi}-\frac{i \psi}{(1+\psi) v_{i}}\left\{\omega^{2}-k_{\perp}^{2} \frac{T_{i 0}}{m_{i}}\right. \\
\left.-k_{\perp}^{2} \frac{T_{e 0}}{m_{i}}\left[1+(1+g) \frac{t_{e 1}}{n_{1}}\right]\right\}=0 .
\end{gathered}
$$

Taking the imaginary part of the dispersion relation gives

$$
\begin{aligned}
\Gamma & -\frac{\psi}{(1+\psi) v_{i}}\left\{\omega_{r}^{2}-k_{\perp}^{2} \frac{T_{i 0}}{m_{i}}\right. \\
& \left.-k_{\perp}^{2} \frac{T_{e 0}}{m_{i}}\left[1+(1+g) \operatorname{Re}\left(\frac{t_{e 1}}{n_{1}}\right)\right]\right\}=0 .
\end{aligned}
$$

Using Eq. (47) in Eq. (51) gives

$$
\begin{aligned}
\Gamma= & \frac{\psi}{(1+\psi) v_{i}}\left\{1+\frac{\psi}{(1+\psi) v_{i}} k_{\perp}^{2} \frac{T_{e 0}}{m_{i}} \xi\right\}^{-1} \\
& \times\left\{\omega_{r}^{2}-k_{\perp}^{2} \frac{T_{i o}}{m_{i}}-k_{\perp}^{2} \frac{T_{e 0}}{m_{i}}\right. \\
& \left.\times\left[1+\frac{(2 / 3)(1+g)^{2}\left(\omega_{r}-\mathbf{k} \cdot \mathbf{V}_{e 0}\right)^{2}}{\left[(2 / 3) \bar{\eta}+k_{\perp}^{2} D_{e}(5+2 g) / 3\right]^{2}+\left(\omega_{r}-\mathbf{k} \cdot \mathbf{V}_{e 0}\right)^{2}}\right]\right\} .
\end{aligned}
$$

Note that the coefficient of $k_{\perp}^{2} T_{e 0} / m_{i}$ in the preceding equation is of the form $1+$ (a positive definite quantity). In other words, the zero growth rate conditions require phase velocities that are greater than the isotropic ion acoustic speed $C_{s}$. As a result, contrary to the traditional view of the thermal diffusion instability where unstable wave behavior is possible even when $\mathbf{V}_{e 0}=0$, we now find that thermal diffusion acts rather as a stabilizing agent. This means, in turn, that even higher $\mathbf{E}_{0} \times \mathbf{B}$ drifts are required than in the pure Farley-Buneman case to drive the plasma unstable.

\section{References}

Dimant, Y. S., and R. N. Sudan, Physical nature of a new crossfield instability in the lower ionosphere, J. Geophys. Res., 102, 25512563, 1997.

Dimant, Y. S., and R. N. Sudan, Kinetic theory of the FarleyBuneman instability in the $\mathrm{E}$ region of the ionosphere, J. Geophys. Res., 100, 14 605-14 623, 1995.

Farley, D. T., and J. Providakes, The variation with $T_{e}$ and $T_{i}$ of the velocity of unstable ionospheric two-stream waves, $J$. Geophys. Res., 94, 15 415-15 420, 1989.

Fejer, B. G., J. Providakes, and D. T. Farley, Theory of plasma waves in the auroral E-region, J. Geophys. Res., 89, 7487-7494, 1984.

Gurevich, A. V., and A. N. Karashtin, Small-scale thermal diffusion instability in the lower ionosphere, Geomagn. Aeron., 24, 733738, 1984.

Hamza, A. M., and J.-P. St.-Maurice, A turbulent theoretical framework for the study of current-driven E region irregularities at high latitudes: basic derivation and application to gradient-free situations, J. Geophys. Res., 98, 11 587-11 599, 1993.

Hirschfelder, J. O., C. F. Curtiss, and R. B. Bird, Molecular theory of gases and liquids, John Wiley, New York, 1954.

Kelley, M. C., The earth's ionosphere, Academic Press, 1989.

Kissack, R. S., J.-P. St.-Maurice, and D. R. Moorcroft, Electron thermal effects on the Farley-Buneman fluid dispersion relation, Phys. Plasmas, 2, 1032-1055, 1995.

Kissack, R. S., J.-P. St.-Maurice, and D. R. Moorcroft, The effect of electron-neutral energy exchange on the fluid FarleyBunenam instability threshold, J. Geophys. Res., 102, 24 091$24115,1997$.

Nielsen, E., and K. Schlegel, A first comparison of STARE and EISCAT electron drift velocity measurements, J. Geophys. Res., 88, 5745-5750, 1983.

Pécseli, H. L., F. Primdahl, and A. Bahnsen, Low-frequency electrostatic turbulence in the polar cap $E$ region, J. Geophys. Res., 94, 5337-5349, 1989.

Robinson, T. R., Towards a self-consistent nonlinear theory of radar auroral backscattering, J. Atmos. Terr. Phys. 48, 417-422, 1986.

Robinson, T. R., The effects of small scale field aligned irregularities on $\mathrm{E}$ region conductivities: implications for electron thermal processes, Adv. Space Sci., 22(9), 1357-1360, 1998.

Schunk, R. W., and A. F. Nagy, Electron temperatures in the F region of the ionosphere: theory and observations, Rev. Geophys. Space Phys., 16, 355-399, 1978.

Schunk, R. W., Mathematical structure of transport equations for multispecies flows, Rev. Geophys. Space Phys., 15, 429-445, 1977.

Shalimov, S., and C. Haldoupis, An electron thermal diffusion instability and type-3 echoes in the auroral E-region plasma, Ann. Geophysicae, 13, 45-55, 1995.

Sudan, R. N., Unified theory of type I and type IV irregularities in the equatorial electrojet, J. Geophys. Res., 88, 4853-4860, 1983. 
St.-Maurice, J.-P., Reply, J. Geophys. Res., 92, 323-327, 1987.

St.-Maurice, J.-P., Electron heating by plasma waves in the high latitude E region and related effects: theory, $A d v$. Space Res., Pergamon Press, 10(6), 239-249, 1990.

St.-Maurice, J.-P., W. Kofman, and E. Kluzek, Electron Heating by plasma waves in the high latitude E-region and related effects: observations, Adv. Space Res., Pergamon Press, 10(6), 225-237, 1990.
St.-Maurice, J.-P., P. Prikryl, D. W. Danskin, A. M. Hamza, G. J. Sofko, J. A. Koehler, A. Kustov, and J. Chen, On the origin of narrow non-ion-acoustic coherent radar spectra in the highlatitude E region, J. Geophys. Res., 99, 6447-6474, 1994.

St.-Maurice, J.-P., C. Cussenot, and W. Kofman, On the usefulness of $E$ region temperatures and lower $F$ region ion temperatures for the extraction of thermospheric paramaters: a case study, Ann. Geophysicae, 17, 1182-1198, 1999. 